\title{
Narrative Ethik als Alternative?
}

\section{Otfried Höffe}

Professor für Philosophie an der Universität Tübingen, Präsident der Nationalen Ethikkommission im Bereich der Humanmedizin
Wer jemanden in Not sieht, fühlt einen Drang zu helfen. Allerdings fühlt den Drang nicht jeder, nach dem bekannten Samaritergleichnis nicht einmal jeder sozial Geachtete, denn der Priester und der Levit lassen den unter die Räuber Gefallenen gleichgültig liegen. Eine narrative Ethik darf daher ihr Ethik-Potential nicht falsch einschätzen. Eine Erzählung wie das Samariter-Gleichnis enthält schon eine moralische Bewertung:

\section{Philosophische Ethik braucht es nur dort, wo das bislang Fraglose grundlegend fragwürdig wird}

Der Priester und der Levit handeln moralisch falsch, der Samariter dagegen moralisch richtig. Dazu kommt die provokative Botschaft: In der für die Menschlichkeit entscheidenden Hinsicht zählen weder die soziale Position («Priester») noch die Stammeszugehörigkeit («Levit»), sondern das, was die Menschen über derartige Unterschiede hinaus vereint: Sie können fremde Not wahrnehmen und sie zu beheben suchen; und im Sinne der häufigen Schlussformel: «Geh' hin und tu desgleichen!» sollen sie es auch.

Glücklicherweise ist das angesichts von Notlagen moralisch Richtige über unsere Kultur- und Epochengrenzen hinweg unstrittig: Allerorten erfährt Hilfs- der Menschheit sprechen [1], freilich erst einem Moralerbe, noch keinem Ethikerbe. Denn «Moral», auch «Moralität» oder «Sittlichkeit», nennen wir den Gegenstand, der wie alle philosophischen Gegenstände von der Philosophie nicht erfunden, sondern vorgefunden wird. «Ethik, auch «Philosophische Ethik» und «Moralphilosophie» heisst dagegen jene methodische Erörterung, die es nur dort braucht, wo das bislang Fraglose, das durch das Vorbild und Nachahmen, durch Lob und Tadel, gegebenenfalls auch Bewunderung oder Verachtung weitergereicht wurde, fragwürdig, sogar grundlegend fragwürdig wird: Die philosophische Ethik bzw. Moralphilosophie entsteht erst in Zeiten von Konflikt, Kritik und Krise. Dann kommt es freilich nicht darauf an, jemandem «die Wahrheit moralischer Urteile argumentativ» anzudemonstrieren, das versuchen allenfalls Fichteaner. Vielmehr gilt es, die Vielfalt der Phänomene auf wenige Begriffe, im Fall einer klaren Gemeinsamkeit sogar auf einen einzigen Begriff zu bringen. Gründliche Philosophen suchen darüber hinaus nach einem veritablen Ursprung, also nach dem, was «Prinzip» im strengen Sinn heisst: nach dem in argumentativer Hinsicht allerersten Anfang. Die Antike, beispielsweise Aristoteles, sieht diesen Anfang im rundum gelungenen Leben, im «eu zēn» bzw. der Eudaimonia, die Moderne in der Freiheit, die, von Kant zu Ende gedacht, in der Autonomie besteht. Darunter ist übrigens nicht irgendeine Selbstbestimmung zu verstehen, sondern deren Radikalgestalt, die Selbstgesetzgebung des Willens.

\section{«Die genaue Art der dem Patienten helfenden, ihm zumindest nicht schadenden Sterbebegleitung mag gelegentlich umstritten sein, die zugrunde liegenden Prinzipien sind es nicht»}

Korrespondenz:

Prof. Dr. phil. Dres. h. c. Otfried Höffe

Philosophisches Seminar Universität Tübingen Bursagasse 1 D-72070 Tübingen sekretariat.hoeffe@ uni-tuebingen.de bereitschaft eine hohe Wertschätzung. Dasselbe trifft auf «Tugenden» wie Besonnenheit und Gerechtigkeit, wie Rechtschaffenheit und Courage zu. Und weil wir selbst einen Grundsatz moralischer Wertschätzung, die Goldene Regel, sowohl in China, etwa bei Konfuzius und Laotse, als auch in Griechenland, bei Thales, finden, ferner im Alten und im Neuen Testament, im indischen Nationalepos Mahabharata und im Buddhismus, nicht zuletzt beim islamischen Denker Al-Ghazali, dürfen wir hier von einem gemeinsamen Moralerbe
Nun kennt die Philosophie für die Reflexion, wenn sie denn aktuell wird, zwei Wege, die Platon im Höhlengleichnis auf dramatische Weise skizziert. Der «Weg hinauf» führt zu den Prinzipien, letztlich dem einen Prinzip, für Platon die Idee des Guten, für Aristoteles die Eudaimonia und für Kant die Autonomie. Dabei ist selbstverständlich der Grundsatz «sōzein ta phainomena» zu beachten. Wird der «Blick auf das Phänomen der Moral» verstellt, so hat der «Weg hinauf» seine Aufgabe verfehlt. 
Den zweiten, nicht alternativen, sondern komplementären Weg, den «Weg hinab», darf man sich nicht als eine formallogische Deduktion vorstellen. Kein Algorithmus, der lediglich mit Situationsbeschreibungen gefüttert wird, führt vom ersten Prinzip zu dem in concreto angemessenen Handeln. Es braucht vielmehr (methodisch komplexe) Beurteilungs- und Vermittlungsprozesse.

\section{Wer für die Bedeutung von Emotionen plädiert, darf asoziale Emotionen wie den Sadismus nicht vergessen, denen man mit Prinzipien wie der Achtung der Menschenwürde entgegentreten darf}

Zusätzlich zur moralischen Fähigkeit ist auch eine ethische Urteilsfähigkeit gefragt. Diese «sagt» zum Beispiel: Wer in einer Notsituation, statt sofort zu helfen, erst einmal sich moralische Prinzipien überlegt, hat beides verkannt - die Moral, die zu helfen gebietet, und die Ethik, die nur dort gefragt ist, wo das Hilfsgebot, zumindest seine Tragweite, so grundsätzlich in Zweifel gezogen wird, wie es vom Samaritergleichnis vorausgesetzt ist. Für den Arzt, zumindest den von heute, stellt sich freilich diese Frage nicht. Denn zu seiner professionellen Aufgabe, dem unantastbaren Berufsethos, gehört es, seinen Patienten zu helfen. Und weil es sich von selbst versteht, würde sich ein Arzt lächerlich machen, wenn er beispielsweise in Fällen von Sterbebegleitung seine (philosophisch gesehen: mittleren) Prinzipien «salus aegroti suprema lex» und «nil nocere» noch ausspricht und mit moralischem Pathos erklärt: Ich wollte dem Patienten helfen, ihm auf keinen Fall schaden. Die genaue Art der dem Patienten helfenden, ihm zumindest nicht schadenden Sterbebegleitung mag gelegentlich umstritten sein, die zugrunde liegenden Prinzipien sind es nicht.

Viele andere Fragen lassen sich aber mit ärztlichem Ethos allein nicht beantworten, so dass es eine veritable, von philosophischen Begriffen und Prinzipien mitgetragene medizinische Ethik braucht.

Dass ein christlicher Theologe narrative Elemente liebt, überrascht den Philosophen nicht. Denn der für den Theologen autoritative Text, das Neue Testament, ist in seinen vier Evangelien reich an Gleichnissen. Diese führen wie beispielsweise das Samaritergleichnis so überzeugende Handlungsmuster vor, dass sie, die Gleichnisse, auch dann ausser dem narrativen einen präskriptiven Charakter haben, wenn die Schlussformel: «Geh hin, und tu desgleichen!» fehlt.

Da Philosophen in der Regel die Moral weder predigen noch zu ihr bekehren, sondern über sie reflektieren wollen, ziehen sie ein anderes genus litterarum vor. Allerdings schliessen sie weder narrative Elemente wie Platons Gleichnisse noch quasi-narrative Elemente wie Gedankenexperimente aus. Um die Wirklichkeit des Sittlichen «dem anderen selbst zu zeigen» fragt im
«Faktum der Vernunft» das Vorbild eines Prinzipienethikers, Kant, ob jemand, der aufgefordert wird, ein falsches Zeugnis wider einen ehrlichen Mann abzulegen, andernfalls aber mit dem Tode bestraft zu werden, es doch für möglich halte, das falsche Zeugnis zu verweigern. Die Antwort auf diese Frage lautet zweifelsohne: ja. Selbst wenn wir das falsche Zeugnis erwarten, da wir mit einem Übergewicht der Selbstliebe rechnen, so halten wir es trotzdem für ein moralisches Unrecht. Um eine solche Beurteilung verstehen zu können, muss man Kant zufolge auf den Begriff eines unbedingten, von einem auch noch so krasser Bedrohung des eigenen Glücks unabhängigen gültigen Gebots, einen kategorischen Imperativ, zurückgreifen [2].

Auch Vertreter der Theologischen Ethik verhalten sich meines Erachtens nicht ganz anders als Moralphilosophen. Geschichten erzählen sie vermutlich nur, wenn sie auf der Kanzel stehen. Halten sie aber Vorlesungen oder Seminare, so prägen sie Begriffe, unterscheiden beispielsweise die Prinzipienethik von einer narrativen Ethik; sie suchen Argumente für und wider und wägen die Argumente gegeneinander ab. Und plädieren sie - wie schon Adam Smith (A Theory of Moral Sentiments) für die Bedeutung von Emotionen, so dürfen sie die asozialen Emotionen wie den Sadismus nicht vergessen, denen man mit Prinzipien wie der Achtung der Freiheit und der Menschenwürde oder dem Verbot der Folter entgegentreten darf.

\section{«Dass ein christlicher Theologe narrative Elemente liebt, überrascht den Philosophen nicht»}

Vielleicht hat Johannes Fischer in meinem Plädoyer gegen die Ethik als Fahne im Wind zu sehr an jenen Teil der Ethik, die Metaethik, gedacht, für die die Haltung des Desengagements konstitutiv ist. Seit meiner Erstlingsschrift «Praktische Philosophie Das Modell des Aristoteles» [3] bis neuerdings zu meiner Fundamentalethik «Lebenskunst und Moral oder Macht Tugend glücklich?» [4] folge ich einem anderen Programm.

\section{Literatur}

1 Vgl. Höffe O (Hrsg.). Lesebuch zur Ethik. Philosophische Texte von der Antike bis zur Gegenwart. München: Beck; 1988, 4. erweiterte Aufl. 2007.

2 Kant I. Kritik der praktischen Vernunft. § 6, Anmerkung.

3 Höffe O. Praktische Philosophie. Das Modell des Aristoteles. Berlin: Akademie Verlag; 1971. 3. Aufl. 2008.

4 Höffe O. Lebenskunst und Moral oder Macht Tugend glücklich? München: C. H. Beck; 2007. Überarbeitetes Taschenbuch; 2009. 